\title{
Generalized Oresme Numbers
}

\author{
Yüksel Soykan
}

\begin{abstract}
Department of Mathematics, Art and Science Faculty,
Zonguldak Bülent Ecevit University, 67100, Zonguldak, Turkey

e-mail: yuksel_soykan@hotmail.com
\end{abstract}

\begin{abstract}
In this paper, we introduce the generalized Oresme sequence and we deal with, in detail, three special cases which we call them modified Oresme, Oresme-Lucas and Oresme sequences. We present Binet's formulas, generating functions, Simson formulas, and the summation formulas for these sequences. Moreover, we give some identities and matrices related with these sequences.
\end{abstract}

\section{Introduction}

The Oresme sequence, $\left\{O_{n}\right\}_{n \geq 0}$, was introduced by Nicole Oresme (1320-1382) in the 14-th century. Oresme found the sum of the rational numbers formed by the terms $0, \frac{1}{2}, \frac{2}{4}, \frac{3}{8}, \frac{4}{16}, \frac{5}{32}, \frac{6}{64}, \ldots, \frac{n}{2^{n}}$. These numbers form a second order sequence and are defined by the recurence relation

$$
O_{n+2}=O_{n+1}-\frac{1}{4} O_{n}, \quad O_{0}=0, O_{1}=\frac{1}{2} .
$$

In [4], Horadam presented a history and obtained an abundance of properties of these numbers. Oresme numbers have many interesting properties and

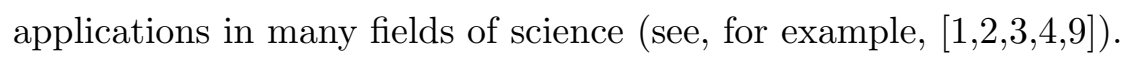

The purpose of this article is to generalize and investigate these interesting sequence of numbers (Oresme numbers). First, we recall some properties of Fibonacci numbers and its generalizations, namely generalized Fibonacci numbers.

Received: August 1, 2021; Accepted: September 5, 2021

2020 Mathematics Subject Classification: 11B37, 11B39, 11 B83.

Keywords and phrases: Oresme numbers, Oresme-Lucas numbers, generalized Fibonacci numbers. 
The Fibonacci numbers, Lucas numbers and their generalizations have many interesting properties and applications to almost every field such as architecture, nature, art, physics and engineering. The sequence of Fibonacci numbers $\left\{F_{n}\right\}_{n \geq 0}$ is defined by

$$
F_{n}=F_{n-1}+F_{n-2}, \quad n \geq 2, \quad F_{0}=0, F_{1}=1,
$$

and the sequence of Lucas numbers $\left\{L_{n}\right\}_{n \geq 0}$ is defined by

$$
L_{n}=L_{n-1}+L_{n-2}, \quad n \geq 2, \quad L_{0}=2, L_{1}=1 .
$$

The generalization of Fibonacci sequence leads to several nice and interesting sequences. The generalized Fibonacci sequence (or generalized $(r, s)$-sequence or Horadam sequence or 2-step Fibonacci sequence) $\left\{W_{n}\left(W_{0}, W_{1} ; r, s\right)\right\}_{n \geq 0}$ (or shortly $\left\{W_{n}\right\}_{n \geq 0}$ ) is defined (by Horadam [6) as follows:

$$
W_{n}=r W_{n-1}+s W_{n-2}, \quad W_{0}=a, W_{1}=b, \quad n \geq 2
$$

where $W_{0}, W_{1}$ are arbitrary complex (or real) numbers and $r, s$ are real numbers, see also Horadam [578 and Soykan [12].

For some specific values of $a, b, r$ and $s$, it is worth presenting these special Horadam numbers in a table as a specific name. In literature, for example, the following names and notations (see Table 1) are used for the special cases of $r, s$ and initial values.

Table 1: A few special case of generalized Fibonacci sequences.

\begin{tabular}{cccc}
\hline Name of sequence & $W_{n}(a, b ; r, s)$ & Binet Formula & OEIS 10] \\
\cline { 2 - 3 } Fibonacci & $W_{n}(0,1 ; 1,1)=F_{n}$ & $\frac{\left(\frac{1+\sqrt{5}}{2}\right)^{n}-\left(\frac{1-\sqrt{5}}{2}\right)^{n}}{\text { A000045 }}$ & A \\
Lucas & $W_{n}(2,1 ; 1,1)=L_{n}$ & $\left(\frac{1+\sqrt{5}}{2}\right)^{n}+\left(\frac{1-\sqrt{5}}{2}\right)^{n}$ & A000032 \\
Pell & $W_{n}(0,1 ; 2,1)=P_{n}$ & $\frac{(1+\sqrt{2})^{n}-(1-\sqrt{2})^{n}}{2 \sqrt{2}}$ & A000129 \\
Pell-Lucas & $W_{n}(2,2 ; 2,1)=Q_{n}$ & $(1+\sqrt{2})^{n}+(1-\sqrt{2})^{n}$ & A002203 \\
Jacobsthal & $W_{n}(0,1 ; 1,2)=J_{n}$ & $\frac{2^{n}-(-1)^{n}}{3}$ & A001045 \\
Jacobsthal-Lucas & $W_{n}(2,1 ; 1,2)=j_{n}$ & $2^{n}+(-1)^{n}$ & A014551 \\
\hline
\end{tabular}


Here, OEIS stands for On-line Encyclopedia of Integer Sequences.

The sequence $\left\{W_{n}\right\}_{n \geq 0}$ can be extended to negative subscripts by defining

$$
W_{-n}=-\frac{r}{s} W_{-(n-1)}+\frac{1}{s} W_{-(n-2)}
$$

for $n=1,2,3, \ldots$ when $s \neq 0$. Therefore, recurrence 1.1 holds for all integer $n$.

Now we define two special cases of the sequence $\left\{W_{n}\right\} . \quad(r, s)$ sequence $\left\{G_{n}(0,1 ; r, s)\right\}_{n \geq 0}$ and Lucas $(r, s)$ sequence $\left\{H_{n}(2, r ; r, s)\right\}_{n \geq 0}$ are defined, respectively, by the second-order recurrence relations

$$
\begin{array}{ll}
G_{n+2}=r G_{n+1}+s G_{n}, & G_{0}=0, G_{1}=1, \\
H_{n+2}=r H_{n+1}+s H_{n}, & H_{0}=2, H_{1}=r,
\end{array}
$$

The sequences $\left\{G_{n}\right\}_{n \geq 0},\left\{H_{n}\right\}_{n \geq 0}$ and $\left\{E_{n}\right\}_{n \geq 0}$ can be extended to negative subscripts by defining

$$
\begin{aligned}
& G_{-n}=-\frac{r}{s} G_{-(n-1)}+\frac{1}{s} G_{-(n-2)}, \\
& H_{-n}=-\frac{r}{s} H_{-(n-1)}+\frac{1}{s} H_{-(n-2)},
\end{aligned}
$$

for $n=1,2,3, \ldots$ respectively. Therefore, recurrences $(1.2)-(1.3)$ hold for all integer $n$.

Some special cases of $(r, s)$ sequence $\left\{G_{n}(0,1 ; r, s)\right\}_{n \geq 0}$ and Lucas $(r, s)$ sequence $\left\{H_{n}(2, r ; r, s)\right\}_{n \geq 0}$ are as follows:

1. $G_{n}(0,1 ; 1,1)=F_{n}$, Fibonacci sequence,

2. $H_{n}(2,1 ; 1,1)=L_{n}$, Lucas sequence,

3. $G_{n}(0,1 ; 2,1)=P_{n}$, Pell sequence,

4. $H_{n}(2,2 ; 2,1)=Q_{n}$, Pell-Lucas sequence,

5. $G_{n}(0,1 ; 1,2)=J_{n}$, Jacobsthal sequence,

6. $H_{n}(2,1 ; 1,2)=j_{n}$, Jacobsthal-Lucas sequence. 
The following theorem shows that the generalized Fibonacci sequence $W_{n}$ at negative indices can be expressed by the sequence itself at positive indices.

Theorem 1. For $n \in \mathbb{Z}$, for the generalized Fibonacci sequence (or generalized $(r, s)$-sequence or Horadam sequence or 2-step Fibonacci sequence), we have the following:

(a)

$$
\begin{aligned}
W_{-n} & =(-1)^{-n-1} s^{-n}\left(W_{n}-H_{n} W_{0}\right) \\
& =(-1)^{n+1} s^{-n}\left(W_{n}-H_{n} W_{0}\right) .
\end{aligned}
$$

(b)

$$
W_{-n}=\frac{(-1)^{n+1} s^{-n}}{-W_{1}^{2}+s W_{0}^{2}+r W_{0} W_{1}}\left(\left(2 W_{1}-r W_{0}\right) W_{0} W_{n+1}-\left(W_{1}^{2}+s W_{0}^{2}\right) W_{n}\right) .
$$

Proof. For the proof, see Soykan [13, Theorem 3.2 and Theorem 3.3].

The following theorem presents sum formulas of generalized $(r, s)$ numbers (generalized Fibonacci numbers).

Theorem 2. Let $x$ be a real (or complex) number. For all integers $m$ and $j$, for generalized $(r, s)$ numbers (generalized Fibonacci numbers), we have the following sum formulas:

(a) If $(-s)^{m} x^{2}-x H_{m}+1 \neq 0$, then

$$
\sum_{k=0}^{n} x^{k} W_{m k+j}=\frac{\begin{array}{c}
\left((-s)^{m} x-H_{m}\right) x^{n+1} W_{m n+j} \\
+(-s)^{m} x^{n+1} W_{m n+j-m}+W_{j}-(-s)^{m} x W_{j-m}
\end{array}}{(-s)^{m} x^{2}-x H_{m}+1} .
$$

(b) If $(-s)^{m} x^{2}-x H_{m}+1=u(x-a)(x-b)=0$ for some $u, a, b \in \mathbb{C}$ with $u \neq 0$ and $a \neq b$, i.e., $x=a$ or $x=b$, then

$$
\sum_{k=0}^{n} x^{k} W_{m k+j}=\frac{\begin{array}{c}
\left(x(n+2)(-s)^{m}-(n+1) H_{m}\right) x^{n} W_{j+m n} \\
+(-s)^{m}(n+1) x^{n} W_{m n+j-m}-(-s)^{m} W_{j-m}
\end{array}}{2(-s)^{m} x-H_{m}} .
$$


(c) If $(-s)^{m} x^{2}-x H_{m}+1=u(x-c)^{2}=0$ for some $u, c \in \mathbb{C}$ with $u \neq 0$, i.e., $x=c$, then

$$
\sum_{k=0}^{n} x^{k} W_{m k+j}=\frac{\begin{array}{c}
(n+1)\left((-s)^{m}(n+2) x^{n}-n x^{n-1} H_{m}\right) W_{m n+j} \\
+n(n+1)(-s)^{m} x^{n-1} W_{m n+j-m}
\end{array}}{2(-s)^{m}} .
$$

Proof. It is given in Soykan [13, Theorem 4.1].

Note that (1.4) can be written in the following form

$$
\sum_{k=1}^{n} x^{k} W_{m k+j}=\frac{\begin{array}{c}
\left((-s)^{m} x-H_{m}\right) x^{n+1} W_{m n+j}+(-s)^{m} x^{n+1} W_{m n+j-m} \\
+x\left(H_{m}-(-s)^{m} x\right) W_{j}-(-s)^{m} x W_{j-m}
\end{array}}{(-s)^{m} x^{2}-x H_{m}+1} .
$$

We give the ordinary generating function $\sum_{n=0}^{\infty} W_{n} x^{n}$ of the sequence $\left\{W_{n}\right\}$.

Lemma 3. Suppose that $f_{W_{n}}(x)=\sum_{n=0}^{\infty} W_{n} x^{n}$ is the ordinary generating function of the generalized Fibonacci sequence $\left\{W_{n}\right\}_{n \geq 0}$. Then, $\sum_{n=0}^{\infty} W_{n} x^{n}$ is given by

$$
\sum_{n=0}^{\infty} W_{n} x^{n}=\frac{W_{0}+\left(W_{1}-r W_{0}\right) x}{1-r x-s x^{2}} .
$$

Proof. For a proof, see [12, Lemma 1.1].

Binet's formula of generalized Fibonacci sequence can be calculated using its characteristic equation (the quadratic equation) which is given as

$$
x^{2}-r x-s=0 .
$$

The roots of characteristic equation are

$$
\alpha=\frac{r+\sqrt{\Delta}}{2}, \beta=\frac{r-\sqrt{\Delta}}{2} .
$$

where

$$
\Delta=r^{2}+4 s
$$


and the followings hold

$$
\begin{aligned}
\alpha+\beta & =r \\
\alpha \beta & =-s, \\
(\alpha-\beta)^{2} & =(\alpha+\beta)^{2}-4 \alpha \beta=r^{2}+4 s .
\end{aligned}
$$

\subsection{Binet's Formula for the Distinct Roots Case}

In this subsection, we assume that the roots $\alpha$ and $\beta$ of characteristic equation (1.6) are distinct. Using these roots and the recurrence relation, Binet's formula can be given as follows:

Theorem 4 (Distinct Roots Case). Binet's formula of generalized Fibonacci numbers is

$$
W_{n}=\frac{b_{1} \alpha^{n}}{\alpha-\beta}+\frac{b_{2} \beta^{n}}{\beta-\alpha}=\frac{b_{1} \alpha^{n}-b_{2} \beta^{n}}{\alpha-\beta}
$$

where

$$
b_{1}=W_{1}-\beta W_{0}, b_{2}=W_{1}-\alpha W_{0} .
$$

(1.8) can be written in the following form:

$$
W_{n}=A_{1} \alpha^{n}+A_{2} \beta^{n}
$$

where

$$
A_{1}=\frac{W_{1}-\beta W_{0}}{\alpha-\beta}, A_{2}=\frac{W_{1}-\alpha W_{0}}{\beta-\alpha} .
$$

Note that

$$
\begin{aligned}
A_{1} A_{2} & =\frac{\left(W_{1}^{2}-s W_{0}^{2}-r W_{1} W_{0}\right)}{-\left(r^{2}+4 s\right)}, \\
A_{1}+A_{2} & =W_{0} .
\end{aligned}
$$

We next find Binet's formula of generalized Fibonacci numbers $\left\{W_{n}\right\}$ by the use of generating function for $W_{n}$. 
Theorem 5 (Binet's formula of generalized Fibonacci numbers).

$$
W_{n}=\frac{d_{1} \alpha^{n}}{(\alpha-\beta)}+\frac{d_{2} \beta^{n}}{(\beta-\alpha)}
$$

where

$$
\begin{aligned}
& d_{1}=W_{0} \alpha+\left(W_{1}-r W_{0}\right), \\
& d_{2}=W_{0} \beta+\left(W_{1}-r W_{0}\right) \beta .
\end{aligned}
$$

Proof. For a proof, see [12, Theorem 1.2].

Note that from (1.8) and 1.10 we have

$$
\begin{aligned}
& W_{1}-\beta W_{0}=W_{0} \alpha+\left(W_{1}-r W_{0}\right), \\
& W_{1}-\alpha W_{0}=W_{0} \beta+\left(W_{1}-r W_{0}\right) \beta .
\end{aligned}
$$

For all integers $n,(r, s)$ and Lucas $(r, s)$ numbers (using initial conditions in (1.8) or 1.10) can be expressed using Binet's formulas as

$$
\begin{aligned}
& G_{n}=\frac{\alpha^{n}}{(\alpha-\beta)}+\frac{\beta^{n}}{(\beta-\alpha)}, \\
& H_{n}=\alpha^{n}+\beta^{n},
\end{aligned}
$$

respectively.

\subsection{Binet's Formula for the Single Root Case}

In this subsection, we assume that the roots $\alpha$ and $\beta$ of characteristic equation (1.6) are equal, i.e., $\alpha=\beta$. So 1.6 can be written as

$$
x^{2}-r x-s=(x-\alpha)^{2}=x^{2}-2 \alpha x+\alpha^{2}=0 .
$$

Note that in this case,

$$
\begin{aligned}
\alpha & =\frac{r}{2}, \\
r & =2 \alpha, \\
s & =-\alpha^{2}=-\frac{r^{2}}{4}, \\
r^{2}+4 s & =0 .
\end{aligned}
$$


Using the root $\alpha$ and the recurrence relation, Binet's formula can be given as follows:

Theorem 6 (Single Root Case). Binet's formula of generalized Fibonacci numbers is

$$
W_{n}=\left(D_{1}+D_{2} n\right) \alpha^{n}
$$

where

$$
\begin{aligned}
D_{1} & =W_{0}, \\
D_{2} & =\frac{1}{\alpha}\left(W_{1}-\alpha W_{0}\right) .
\end{aligned}
$$

Proof. For a proof, see Soykan 13.

Note that 1.13 can be written as

$$
\begin{aligned}
W_{n} & =\left(W_{0}+\frac{1}{\alpha}\left(W_{1}-\alpha W_{0}\right) n\right) \alpha^{n} \\
& =\left(n W_{1}-\alpha(n-1) W_{0}\right) \alpha^{n-1} \\
& =\left(n W_{1}-\frac{r}{2}(n-1) W_{0}\right)\left(\frac{r}{2}\right)^{n-1} .
\end{aligned}
$$

We also see that

$$
\begin{aligned}
D_{1} D_{2} & =\frac{W_{0}\left(2 W_{1}-r W_{0}\right)}{r}, \\
D_{1}+D_{2} & =2 \frac{W_{1}}{r} .
\end{aligned}
$$

For all integers $n,(r, s)$ and Lucas $(r, s)$ numbers (using initial conditions in (1.8) or 1.10$)$ ) can be expressed using Binet's formulas as

$$
\begin{aligned}
& G_{n}=n \alpha^{n-1}, \\
& H_{n}=2 \alpha^{n},
\end{aligned}
$$

respectively. 


\section{Generalized Oresme Sequence}

In this paper we consider the case $r=1, s=-\frac{1}{4}$. A generalized Oresme sequence $\left\{W_{n}\right\}_{n \geq 0}=\left\{W_{n}\left(W_{0}, W_{1}\right)\right\}_{n \geq 0}$ is defined by the second-order recurrence relations

$$
W_{n}=W_{n-1}-\frac{1}{4} W_{n-2}
$$

with the initial values $W_{0}=c_{0}, W_{1}=c_{1}$ not all being zero.

The sequence $\left\{W_{n}\right\}_{n \geq 0}$ can be extended to negative subscripts by defining

$$
W_{-n}=4 W_{-(n-1)}-4 W_{-(n-2)}
$$

for $n=1,2,3, \ldots$. Therefore, recurrence (2.1) holds for all integers $n$.

Eq. 1.13 can be used to obtain Binet formula of generalized Oresme numbers. Binet formula of generalized Oresme numbers can be given as

$$
W_{n}=\left(D_{1}+D_{2} n\right) \alpha^{n}
$$

where

$$
\begin{aligned}
D_{1} & =W_{0}, \\
D_{2} & =\frac{1}{\alpha}\left(W_{1}-\alpha W_{0}\right) .
\end{aligned}
$$

i.e.,

$$
W_{n}=\left(W_{0}+\frac{1}{\alpha}\left(W_{1}-\alpha W_{0}\right) n\right) \alpha^{n} .
$$

Here, $\alpha=\beta=\frac{1}{2}$ are the roots of the quadratic equation

$$
x^{2}-x+\frac{1}{4}=0 .
$$

i.e. the roots of characteristic equation $(2.3)$ are equal. Note that

$$
\begin{aligned}
\alpha+\beta & =1, \\
\alpha \beta & =\frac{1}{4}, \\
\alpha-\beta & =0 .
\end{aligned}
$$


and

$$
W_{n}=\left(W_{0}+2\left(W_{1}-\frac{1}{2} W_{0}\right) n\right) \times \frac{1}{2^{n}} .
$$

The first few generalized Oresme numbers with positive subscript and negative subscript are given in the following Table 2 .

Table 2: A few generalized Oresme numbers.

\begin{tabular}{ccc}
\hline$n$ & $W_{n}$ & $W_{-n}$ \\
\hline 0 & $W_{0}$ & $W_{0}$ \\
1 & $W_{1}$ & $4 W_{0}-4 W_{1}$ \\
2 & $W_{1}-\frac{1}{4} W_{0}$ & $12 W_{0}-16 W_{1}$ \\
3 & $\frac{3}{4} W_{1}-\frac{1}{4} W_{0}$ & $32 W_{0}-48 W_{1}$ \\
4 & $\frac{1}{2} W_{1}-\frac{3}{16} W_{0}$ & $80 W_{0}-128 W_{1}$ \\
5 & $\frac{5}{16} W_{1}-\frac{1}{8} W_{0}$ & $192 W_{0}-320 W_{1}$ \\
6 & $\frac{3}{16} W_{1}-\frac{5}{64} W_{0}$ & $448 W_{0}-768 W_{1}$ \\
7 & $\frac{7}{64} W_{1}-\frac{3}{64} W_{0}$ & $1024 W_{0}-1792 W_{1}$ \\
8 & $\frac{1}{16} W_{1}-\frac{7}{256} W_{0}$ & $2304 W_{0}-4096 W_{1}$ \\
9 & $\frac{9}{256} W_{1}-\frac{1}{64} W_{0}$ & $5120 W_{0}-9216 W_{1}$ \\
10 & $\frac{5}{256} W_{1}-\frac{9}{1024} W_{0}$ & $11264 W_{0}-20480 W_{1}$ \\
\hline
\end{tabular}

Now we define three special cases of the sequence $\left\{W_{n}\right\}$. Modified Oresme sequence $\left\{G_{n}\right\}_{n \geq 0}$, Oresme-Lucas sequence $\left\{H_{n}\right\}_{n \geq 0}$ and Oresme sequence $\left\{O_{n}\right\}_{n \geq 0}$ are defined, respectively, by the second-order recurrence relations

$$
\begin{array}{ll}
G_{n+2}=G_{n+1}-\frac{1}{4} G_{n}, & G_{0}=0, G_{1}=1, \\
H_{n+2}=H_{n+1}-\frac{1}{4} H_{n}, & H_{0}=2, H_{1}=1, \\
O_{n+2}=O_{n+1}-\frac{1}{4} O_{n}, & O_{0}=0, O_{1}=\frac{1}{2} .
\end{array}
$$

The sequences $\left\{G_{n}\right\}_{n \geq 0},\left\{H_{n}\right\}_{n \geq 0}$ and $\left\{O_{n}\right\}_{n \geq 0}$ can be extended to negative 
subscripts by defining

$$
\begin{aligned}
& G_{-n}=4 G_{-(n-1)}-4 G_{-(n-2)}, \\
& H_{-n}=4 H_{-(n-1)}-4 H_{-(n-2)}, \\
& O_{-n}=4 O_{-(n-1)}-4 O_{-(n-2)},
\end{aligned}
$$

for $n=1,2,3, \ldots$ respectively. Therefore, recurrences (2.4)-(2.6) hold for all integers $n$.

Next, we present the first few values of the modified Oresme, Oresme-Lucas and Oresme numbers with positive and negative subscripts:

Table 3: The first few values of the special second-order numbers with positive and negative subscripts.

\begin{tabular}{ccccccccccccc}
\hline$n$ & 0 & 1 & 2 & 3 & 4 & 5 & 6 & 7 & 8 & 9 & 10 & 11 \\
\hline$G_{n}$ & 0 & 1 & 1 & $\frac{3}{4}$ & $\frac{1}{2}$ & $\frac{5}{16}$ & $\frac{3}{16}$ & $\frac{7}{64}$ & $\frac{1}{16}$ & $\frac{9}{256}$ & $\frac{5}{256}$ & $\frac{11}{1024}$ \\
$G_{-n}$ & $\ldots$ & -4 & -16 & -48 & -128 & -320 & -768 & -1792 & -4096 & -9216 & -20480 & -45056 \\
$H_{n}$ & 2 & 1 & $\frac{1}{2}$ & $\frac{1}{4}$ & $\frac{1}{8}$ & $\frac{1}{16}$ & $\frac{1}{32}$ & $\frac{1}{64}$ & $\frac{1}{128}$ & $\frac{1}{256}$ & $\frac{1}{512}$ & $\frac{1}{1024}$ \\
$H_{-n}$ & $\ldots$ & 4 & 8 & 16 & 32 & 64 & 128 & 256 & 512 & 1024 & 2048 & 4096 \\
$O_{n}$ & 0 & $\frac{1}{2}$ & $\frac{1}{2}$ & $\frac{3}{8}$ & $\frac{1}{4}$ & $\frac{5}{32}$ & $\frac{3}{32}$ & $\frac{7}{128}$ & $\frac{1}{32}$ & $\frac{9}{512}$ & $\frac{5}{512}$ & $\frac{11}{2048}$ \\
$O_{-n}$ & $\ldots$ & -2 & -8 & -24 & -64 & -160 & -384 & -896 & -2048 & -4608 & -10240 & -22528 \\
\hline
\end{tabular}

For all integers $n$, modified Oresme, Oresme-Lucas and Oresme numbers (using initial conditions in (2.2) ) can be expressed using Binet's formulas as

$$
\begin{aligned}
& G_{n}=n \alpha^{n-1}=\frac{n}{2^{n-1}} \\
& H_{n}=2 \alpha^{n}=\frac{1}{2^{n-1}}, \\
& O_{n}=n \alpha^{n}=\frac{n}{2^{n}}
\end{aligned}
$$

respectively. 
Note that

$$
\begin{aligned}
G_{n} & =2 O_{n}, \\
G_{n} & =\frac{n}{2^{n-1}}=n H_{n}, \\
O_{n} & =\frac{n}{2^{n}}=\frac{n}{2} H_{n} .
\end{aligned}
$$

Next, we give the ordinary generating function $\sum_{n=0}^{\infty} W_{n} x^{n}$ of the sequence $\left\{W_{n}\right\}$.

Lemma 7. Suppose that $f_{W_{n}}(x)=\sum_{n=0}^{\infty} W_{n} x^{n}$ is the ordinary generating function of the generalized Oresme sequence $\left\{W_{n}\right\}_{n \geq 0}$. Then, $\sum_{n=0}^{\infty} W_{n} x^{n}$ is given by

$$
\sum_{n=0}^{\infty} W_{n} x^{n}=4 \times \frac{W_{0}+\left(W_{1}-W_{0}\right) x}{(x-2)^{2}} .
$$

Proof. In Lemma 3, take $r=1, s=-\frac{1}{4}$.

The previous Lemma gives the following results as particular examples.

Corollary 8. Generated functions of modified Oresme, Oresme-Lucas and Oresme numbers are

$$
\begin{aligned}
\sum_{n=0}^{\infty} G_{n} x^{n} & =\frac{4 x}{(x-2)^{2}}, \\
\sum_{n=0}^{\infty} H_{n} x^{n} & =-\frac{4}{x-2}, \\
\sum_{n=0}^{\infty} O_{n} x^{n} & =\frac{2 x}{(x-2)^{2}},
\end{aligned}
$$

respectively.

Proof. In Lemma 7, take $W_{n}=G_{n}$ with $G_{0}=0, G_{1}=1, W_{n}=H_{n}$ with $H_{0}=$ $2, H_{1}=1$ and $W_{n}=O_{n}$ with $O_{0}=0, O_{1}=\frac{1}{2}$, respectively. 


\section{Simson Formulas}

There is a well-known Simson Identity (formula) for Fibonacci sequence $\left\{F_{n}\right\}$, namely,

$$
F_{n+1} F_{n-1}-F_{n}^{2}=(-1)^{n}
$$

which was derived first by R. Simson in 1753 and it is now called as Cassini Identity (formula) as well. This can be written in the form

$$
\left|\begin{array}{cc}
F_{n+1} & F_{n} \\
F_{n} & F_{n-1}
\end{array}\right|=(-1)^{n} .
$$

The following theorem gives generalization of this result to the generalized Oresme sequence $\left\{W_{n}\right\}_{n \geq 0}$.

Theorem 9 (Simson Formula of Generalized Oresme Numbers). For all integers $n$, we have

$$
\left|\begin{array}{cc}
W_{n+1} & W_{n} \\
W_{n} & W_{n-1}
\end{array}\right|=\left(\frac{1}{4}\right)^{n}\left|\begin{array}{cc}
W_{1} & W_{0} \\
W_{0} & W_{-1}
\end{array}\right| .
$$

Proof. For a proof of Eq. 3.1, see Soykan 11, just take $s=-\frac{1}{4}$.

The previous theorem gives the following results as particular examples.

Corollary 10. For all integers n, modified Oresme, Oresme-Lucas and Oresme numbers are given as

$$
\begin{aligned}
\left|\begin{array}{cc}
G_{n+1} & G_{n} \\
G_{n} & G_{n-1}
\end{array}\right| & =\frac{-1}{4^{n-1}}, \\
\left|\begin{array}{cc}
H_{n+1} & H_{n} \\
H_{n} & H_{n-1}
\end{array}\right| & =0, \\
\left|\begin{array}{cc}
O_{n+1} & O_{n} \\
O_{n} & O_{n-1}
\end{array}\right| & =\frac{-1}{4^{n}},
\end{aligned}
$$

respectively. 


\section{Some Identities}

In this section, we obtain some identities of generalized Oresme, modified Oresme, Oresme-Lucas and Oresme numbers. First, we can give a few basic relations between $\left\{W_{n}\right\}$ and $\left\{G_{n}\right\}$.

Lemma 11. The following equalities are true:

$$
\begin{aligned}
& W_{n}=16\left(2 W_{0}-3 W_{1}\right) G_{n+4}-4\left(5 W_{0}-8 W_{1}\right) G_{n+3}, \\
& W_{n}=4\left(3 W_{0}-4 W_{1}\right) G_{n+3}-4\left(2 W_{0}-3 W_{1}\right) G_{n+2}, \\
& W_{n}=4\left(W_{0}-W_{1}\right) G_{n+2}-\left(3 W_{0}-4 W_{1}\right) G_{n+1}, \\
& W_{n}=W_{0} G_{n+1}-\left(W_{0}-W_{1}\right) G_{n}, \\
& W_{n}=W_{1} G_{n}-\frac{1}{4} W_{0} G_{n-1},
\end{aligned}
$$

and

$$
\begin{aligned}
& \left(W_{0}-2 W_{1}\right)^{2} G_{n}=64\left(W_{0}-3 W_{1}\right) W_{n+4}-16\left(3 W_{0}-8 W_{1}\right) W_{n+3}, \\
& \left(W_{0}-2 W_{1}\right)^{2} G_{n}=16\left(W_{0}-4 W_{1}\right) W_{n+3}-16\left(W_{0}-3 W_{1}\right) W_{n+2}, \\
& \left(W_{0}-2 W_{1}\right)^{2} G_{n}=-16 W_{1} W_{n+2}-4\left(W_{0}-4 W_{1}\right) W_{n+1}, \\
& \left(W_{0}-2 W_{1}\right)^{2} G_{n}=-4 W_{0} W_{n+1}+4 W_{1} W_{n} \\
& \left(W_{0}-2 W_{1}\right)^{2} G_{n}=-4\left(W_{0}-W_{1}\right) W_{n}+W_{0} W_{n-1} .
\end{aligned}
$$

Proof. Note that all the identities hold for all integers $n$. We prove 4.1). To show (4.1), writing

$$
W_{n}=a \times G_{n+4}+b \times G_{n+3}
$$

and solving the system of equations

$$
\begin{aligned}
& W_{0}=a \times G_{4}+b \times G_{3} \\
& W_{1}=a \times G_{5}+b \times G_{4}
\end{aligned}
$$

we find that $a=16\left(2 W_{0}-3 W_{1}\right), b=-4\left(5 W_{0}-8 W_{1}\right)$. The other equalities can be proved similarly. 
Note that all the identities in the above Lemma can be proved by induction as well.

Next, we present a few basic relations between $\left\{H_{n}\right\}$ and $\left\{W_{n}\right\}$.

Lemma 12. The following equalities are true:

$$
\begin{aligned}
& \left(W_{0}-2 W_{1}\right) H_{n}=-32 W_{n+4}+16 W_{n+3}, \\
& \left(W_{0}-2 W_{1}\right) H_{n}=-16 W_{n+3}+8 W_{n+2}, \\
& \left(W_{0}-2 W_{1}\right) H_{n}=-8 W_{n+2}+4 W_{n+1}, \\
& \left(W_{0}-2 W_{1}\right) H_{n}=-4 W_{n+1}+2 W_{n}, \\
& \left(W_{0}-2 W_{1}\right) H_{n}=-2 W_{n}+W_{n-1} .
\end{aligned}
$$

Now, we give a few basic relations between $\left\{W_{n}\right\}$ and $\left\{O_{n}\right\}$.

Lemma 13. The following equalities are true:

$$
\begin{aligned}
& W_{n}=32\left(2 W_{0}-3 W_{1}\right) O_{n+4}-8\left(5 W_{0}-8 W_{1}\right) O_{n+3}, \\
& W_{n}=8\left(3 W_{0}-4 W_{1}\right) O_{n+3}-8\left(2 W_{0}-3 W_{1}\right) O_{n+2}, \\
& W_{n}=8\left(W_{0}-W_{1}\right) O_{n+2}-2\left(3 W_{0}-4 W_{1}\right) O_{n+1}, \\
& W_{n}=2 W_{0} O_{n+1}-2\left(W_{0}-W_{1}\right) O_{n}, \\
& W_{n}=2 W_{1} O_{n}-\frac{1}{2} W_{0} O_{n-1},
\end{aligned}
$$

and

$$
\begin{aligned}
& \left(W_{0}-2 W_{1}\right)^{2} O_{n}=32\left(W_{0}-3 W_{1}\right) W_{n+4}-8\left(3 W_{0}-8 W_{1}\right) W_{n+3}, \\
& \left(W_{0}-2 W_{1}\right)^{2} O_{n}=8\left(W_{0}-4 W_{1}\right) W_{n+3}-8\left(W_{0}-3 W_{1}\right) W_{n+2}, \\
& \left(W_{0}-2 W_{1}\right)^{2} O_{n}=-8 W_{1} W_{n+2}-2\left(W_{0}-4 W_{1}\right) W_{n+1}, \\
& \left(W_{0}-2 W_{1}\right)^{2} O_{n}=-2 W_{0} W_{n+1}+2 W_{1} W_{n}, \\
& \left(W_{0}-2 W_{1}\right)^{2} O_{n}=-2\left(W_{0}-W_{1}\right) W_{n}+\frac{1}{2} W_{0} W_{n-1} .
\end{aligned}
$$

Now, we give a few basic relations between $\left\{G_{n}\right\},\left\{H_{n}\right\}$ and $\left\{O_{n}\right\}$. 
Lemma 14. The following equalities are true:

$$
\begin{aligned}
& H_{n}=16 G_{n+4}-8 G_{n+3} \\
& H_{n}=8 G_{n+3}-4 G_{n+2} \\
& H_{n}=4 G_{n+2}-2 G_{n+1} \\
& H_{n}=2 G_{n+1}-G_{n} \\
& H_{n}=G_{n}-\frac{1}{2} G_{n-1}
\end{aligned}
$$

and

$$
\begin{aligned}
O_{n} & =-24 G_{n+4}+16 G_{n+3}, \\
O_{n} & =-8 G_{n+3}+6 G_{n+2}, \\
O_{n} & =-2 G_{n+2}+2 G_{n+1}, \\
O_{n} & =\frac{1}{2} G_{n}
\end{aligned}
$$

and

$$
\begin{aligned}
G_{n} & =-96 O_{n+4}+64 O_{n+3}, \\
G_{n} & =-32 O_{n+3}+24 O_{n+2}, \\
G_{n} & =-8 O_{n+2}+8 O_{n+1}, \\
G_{n} & =2 O_{n},
\end{aligned}
$$

and

$$
\begin{aligned}
& H_{n}=32 O_{n+4}-16 O_{n+3}, \\
& H_{n}=16 O_{n+3}-8 O_{n+2}, \\
& H_{n}=8 O_{n+2}-4 O_{n+1}, \\
& H_{n}=4 O_{n+1}-2 O_{n}, \\
& H_{n}=2 O_{n}-O_{n-1},
\end{aligned}
$$

and

$$
\begin{aligned}
G_{n} & =\frac{n}{2^{n-1}}=n H_{n} \\
O_{n} & =\frac{n}{2^{n}}=\frac{n}{2} H_{n} .
\end{aligned}
$$


We now present a few special identities for the generalized Oresme sequence $\left\{W_{n}\right\}$.

Theorem 15 (Catalan's identity of the generalized Oresme sequence). For all integers $n$ and $m$, the following identity holds:

$$
W_{n+m} W_{n-m}-W_{n}^{2}=-\frac{m^{2}}{2^{2 n}}\left(W_{0}-2 W_{1}\right)^{2} .
$$

Proof. We use the identity

$$
W_{n}=\left(W_{0}+2\left(W_{1}-\frac{1}{2} W_{0}\right) n\right) \times \frac{1}{2^{n}} .
$$

As special cases of the above theorem, we have the following corollary.

Corollary 16. For all integers $n$ and $m$, the following identities hold:

(a) $G_{n+m} G_{n-m}-G_{n}^{2}=-\frac{m^{2}}{2^{2 n-2}}$.

(b) $H_{n+m} H_{n-m}-H_{n}^{2}=0$.

(c) $O_{n+m} O_{n-m}-O_{n}^{2}=-\frac{m^{2}}{2^{2 n}}$.

Note that for $m=1$ in Catalan's identity of the generalized Oresme sequence, we get the Cassini identity for the generalized Oresme sequnce.

Theorem 17 (Cassini's identity of the generalized Oresme sequence). For all integers $n$, the following identity holds:

$$
W_{n+1} W_{n-1}-W_{n}^{2}=-\frac{1}{2^{2 n}}\left(W_{0}-2 W_{1}\right)^{2} .
$$

As special cases of the above theorem, we have the following corollary.

Corollary 18. For all integers n, the following identities hold:

(a) $G_{n+1} G_{n-1}-G_{n}^{2}=-\frac{1}{2^{2 n-2}}$. 
(b) $H_{n+1} H_{n-1}-H_{n}^{2}=0$.

(c) $O_{n+1} O_{n-1}-O_{n}^{2}=-\frac{1}{2^{2 n}}$.

The d'Ocagne's, Gelin-Cesàro's and Melham' identities can also be obtained by using

$$
W_{n}=\left(W_{0}+2\left(W_{1}-\frac{1}{2} W_{0}\right) n\right) \times \frac{1}{2^{n}} .
$$

The next theorem presents d'Ocagne's, Gelin-Cesàro's and Melham' identities of generalized Oresme sequence $\left\{W_{n}\right\}$.

Theorem 19. Let $n$ and $m$ be any integers. Then the following identities are true:

(a) (d'Ocagne's identity)

$$
W_{m+1} W_{n}-W_{m} W_{n+1}=-\frac{(m-n)}{2^{m+n+1}}\left(W_{0}-2 W_{1}\right)^{2} .
$$

(b) (Gelin-Cesàro's identity)

$$
\begin{aligned}
& W_{n+2} W_{n+1} W_{n-1} W_{n-2}-W_{n}^{4} \\
= & -\frac{1}{2^{4 n}}\left(4\left(5 n^{2}-4\right) W_{1}^{2}+\left(5 n^{2}-10 n+1\right) W_{0}^{2}-4\left(5 n^{2}-5 n-4\right) W_{1} W_{0}\right)\left(W_{0}-2 W_{1}\right)^{2} .
\end{aligned}
$$

(c) (Melham's identity)

$$
W_{n+1} W_{n+2} W_{n+6}-W_{n+3}^{3}=-\frac{1}{2^{3 n+9}}\left(2(7 n+15) W_{1}-(7 n+8) W_{0}\right)\left(W_{0}-2 W_{1}\right)^{2} .
$$

Proof. Use the identity $W_{n}=\left(W_{0}+2\left(W_{1}-\frac{1}{2} W_{0}\right) n\right) \times \frac{1}{2^{n}}$.

As special cases of the above theorem, we have the following three corollaries. First one presents d'Ocagne's, Gelin-Cesàro's and Melham' identities of modified Oresme sequence $\left\{G_{n}\right\}$.

Corollary 20. Let $n$ and $m$ be any integers. Then the following identities are true: 
(a) (d'Ocagne's identity)

$$
G_{m+1} G_{n}-G_{m} G_{n+1}=-\frac{(m-n)}{2^{m+n-1}} .
$$

(b) (Gelin-Cesàro's identity)

$$
G_{n+2} G_{n+1} G_{n-1} G_{n-2}-G_{n}^{4}=-\frac{\left(5 n^{2}-4\right)}{2^{4 n-4}} .
$$

(c) (Melham's identity)

$$
G_{n+1} G_{n+2} G_{n+6}-G_{n+3}^{3}=-\frac{(7 n+15)}{2^{3 n+6}} .
$$

Second one presents d'Ocagne's, Gelin-Cesàro's and Melham' identities of Oresme-Lucas sequence $\left\{H_{n}\right\}$.

Corollary 21. Let $n$ and $m$ be any integers. Then the following identities are true:

(a) (d'Ocagne's identity)

$$
H_{m+1} H_{n}-H_{m} H_{n+1}=0 .
$$

(b) (Gelin-Cesàro's identity)

$$
H_{n+2} H_{n+1} H_{n-1} H_{n-2}-H_{n}^{4}=0 .
$$

(c) (Melham's identity)

$$
H_{n+1} H_{n+2} H_{n+6}-H_{n+3}^{3}=0 .
$$

Third one presents d'Ocagne's, Gelin-Cesàro's and Melham' identities of Oresme sequence $\left\{O_{n}\right\}$.

Corollary 22. Let $n$ and $m$ be any integers. Then the following identities are true: 
(a) (d'Ocagne's identity)

$$
O_{m+1} O_{n}-O_{m} O_{n+1}=-\frac{(m-n)}{2^{m+n+1}} .
$$

(b) (Gelin-Cesàro's identity)

$$
O_{n+2} O_{n+1} O_{n-1} O_{n-2}-O_{n}^{4}=-\frac{\left(5 n^{2}-4\right)}{2^{4 n}} .
$$

(c) (Melham's identity)

$$
O_{n+1} O_{n+2} O_{n+6}-O_{n+3}^{3}=-\frac{(7 n+15)}{2^{3 n+9}} .
$$

\section{On the Recurrence Properties of Generalized Oresme Sequence}

Taking $r=1, s=-\frac{1}{4}$ in Theorem 1 (a) and (b), we obtain the following Proposition.

Proposition 23. For $n \in \mathbb{Z}$, generalized Oresme numbers (the case $r=1, s=$ $-\frac{1}{4}$ ) have the following identity:

$$
\begin{aligned}
W_{-n} & =(-1)^{n+1}\left(-\frac{1}{4}\right)^{-n}\left(W_{n}-H_{n} W_{0}\right) \\
& =\frac{(-1)^{n+1}\left(-\frac{1}{4}\right)^{-n}}{-W_{1}^{2}-\frac{1}{4} W_{0}^{2}+W_{0} W_{1}}\left(\left(2 W_{1}-W_{0}\right) W_{0} W_{n+1}-\left(W_{1}^{2}-\frac{1}{4} W_{0}^{2}\right) W_{n}\right) .
\end{aligned}
$$

From the above Proposition, we have the following corollary which gives the connection between the special cases of generalized Oresme sequence at the positive index and the negative index: for modified Oresme, Oresme-Lucas and Oresme numbers: take $W_{n}=G_{n}$ with $G_{0}=0, G_{1}=1$, take $W_{n}=H_{n}$ with $H_{0}=2, H_{1}=1$ and $W_{n}=O_{n}$ with $O_{0}=0, O_{1}=\frac{1}{2}$, respectively. Note that in this case $H_{n}=H_{n}$.

Corollary 24. For $n \in \mathbb{Z}$, we have the following recurrence relations: 
(a) modified Oresme sequence:

$$
G_{-n}=-4^{n} G_{n}=-n \times 2^{n+1} .
$$

(b) Oresme-Lucas sequence:

$$
H_{-n}=4^{n} H_{n}=2^{n+1} \text {. }
$$

(c) Oresme sequence:

$$
O_{-n}=-4^{n} O_{n}=-n \times 2^{n} .
$$

\section{The Sum Formula $\sum_{k=0}^{n} x^{k} W_{m k+j}$ of Generalized Oresme Numbers}

In this section, we present sum formulas of generalized Oresme numbers.

Theorem 25. Let $x$ be a real (or complex) number. For all integers $m$ and $j$, for generalized Oresme numbers, we have the following sum formulas:

(a) If $2^{-2 m} x^{2}-x H_{m}+1 \neq 0 \neq 0$, then

$$
\sum_{k=0}^{n} x^{k} W_{m k+j}=\frac{\left(\begin{array}{c}
\left.2^{-2 m} x-H_{m}\right) x^{n+1} W_{m n+j} \\
+2^{-2 m} x^{n+1} W_{m n+j-m}+W_{j}-2^{-2 m} x W_{j-m}
\end{array}\right.}{2^{-2 m} x^{2}-x H_{m}+1} .
$$

(b) If $2^{-2 m} x^{2}-x H_{m}+1=u(x-a)(x-b)=0$ for some $u, a, b \in \mathbb{C}$ with $u \neq 0$ and $a \neq b$, i.e., $x=a$ or $x=b$, then

$$
\sum_{k=0}^{n} x^{k} W_{m k+j}=\frac{\begin{array}{c}
\left(x(n+2) 2^{-2 m}-(n+1) H_{m}\right) x^{n} W_{j+m n} \\
+2^{-2 m}(n+1) x^{n} W_{m n+j-m}-2^{-2 m} W_{j-m}
\end{array}}{2^{-2 m+1} x-H_{m}} .
$$

(c) If $2^{-2 m} x^{2}-x H_{m}+1=u(x-c)^{2}=0$ for some $u, c \in \mathbb{C}$ with $u \neq 0$, i.e., $x=c$, then

$$
\sum_{k=0}^{n} x^{k} W_{m k+j}=\frac{\begin{array}{c}
(n+1)\left(2^{-2 m}(n+2) x^{n}-n x^{n-1} H_{m}\right) W_{m n+j} \\
+n(n+1) 2^{-2 m} x^{n-1} W_{m n+j-m}
\end{array}}{2^{-2 m+1}} .
$$


Proof. Take $r=1, s=-\frac{1}{4}$ and $H_{n}=H_{n}$ in Theorem 2 .

Note that $(6.1)$ can be written in the following form

$$
\sum_{k=1}^{n} x^{k} W_{m k+j}=\frac{\begin{array}{c}
\left(2^{-2 m} x-H_{m}\right) x^{n+1} W_{m n+j}+2^{-2 m} x^{n+1} W_{m n+j-m} \\
+x\left(H_{m}-2^{-2 m} x\right) W_{j}-2^{-2 m} x W_{j-m}
\end{array}}{2^{-2 m} x^{2}-x H_{m}+1} .
$$

As special cases of $m$ and $j$ in the last Theorem, we obtain the following proposition.

Proposition 26. For generalized Oresme numbers, we have the following sum formulas:

(a) $(m=1, j=0)$

If $\frac{1}{4}(x-2)^{2} \neq 0$, i.e., $x \neq 2$, then

$$
\sum_{k=0}^{n} x^{k} W_{k}=\frac{(x-4) x^{n+1} W_{n}+x^{n+1} W_{n-1}+4 W_{0}+4\left(W_{1}-W_{0}\right) x}{(x-2)^{2}},
$$

and

$$
\begin{aligned}
& \text { if } \frac{1}{4}(x-2)^{2}=0 \text {, i.e., } x=2 \text {, then } \\
& \qquad \sum_{k=0}^{n} x^{k} W_{k}=\frac{(n+1)((x-4) n+2 x) x^{n-1} W_{n}+n(n+1) x^{n-1} W_{n-1}}{2} .
\end{aligned}
$$

(b) $(m=2, j=0)$

If $\frac{1}{16}(x-4)^{2} \neq 0$, i.e., $x \neq 4$, then

$$
\sum_{k=0}^{n} x^{k} W_{2 k}=\frac{(x-8) x^{n+1} W_{2 n}+x^{n+1} W_{2 n-2}+16 W_{0}+4\left(4 W_{1}-3 W_{0}\right) x}{(x-4)^{2}},
$$

and

if $\frac{1}{16}(x-4)^{2}=0$, i.e., $x=4$, then

$$
\sum_{k=0}^{n} x^{k} W_{2 k}=\frac{(n+1)((x-8) n+2 x) x^{n-1} W_{2 n}+n(n+1) x^{n-1} W_{2 n-2}}{2} .
$$


(c) $(m=2, j=1)$

If $\frac{1}{16}(x-4)^{2} \neq 0$, i.e., $x \neq 4$, then

$\sum_{k=0}^{n} x^{k} W_{2 k+1}=\frac{(x-8) x^{n+1} W_{2 n+1}+x^{n+1} W_{2 n-1}+16 W_{1}+4\left(W_{1}-W_{0}\right) x}{(x-4)^{2}}$,

and

if $\frac{1}{16}(x-4)^{2}=0$, i.e., $x=4$, then

$\sum_{k=0}^{n} x^{k} W_{2 k+1}=\frac{(n+1)((x-8) n+2 x) x^{n-1} W_{2 n+1}+n(n+1) x^{n-1} W_{2 n-1}}{2}$.

(d) $(m=-1, j=0)$

If $(2 x-1)^{2} \neq 0$, i.e., $x \neq \frac{1}{2}$, then

$$
\sum_{k=0}^{n} x^{k} W_{-k}=\frac{4 x^{n+1} W_{-n+1}+4(x-1) x^{n+1} W_{-n}+W_{0}-4 x W_{1}}{(2 x-1)^{2}},
$$

and

if $(2 x-1)^{2}=0$, i.e., $x=\frac{1}{2}$, then

$$
\sum_{k=0}^{n} x^{k} W_{-k}=\frac{n(n+1) x^{n-1} W_{-n+1}+(n+1)((x-1) n+2 x) x^{n-1} W_{-n}}{2} .
$$

(e) $(m=-2, j=0)$

If $(4 x-1)^{2} \neq 0$, i.e., $x \neq \frac{1}{4}$, then

$\sum_{k=0}^{n} x^{k} W_{-2 k}=\frac{16 x^{n+1} W_{-2 n+2}+8(2 x-1) x^{n+1} W_{-2 n}+W_{0}-4\left(4 W_{1}-W_{0}\right) x}{(4 x-1)^{2}}$,

and

if $(4 x-1)^{2}=0$, i.e., $x=\frac{1}{4}$, then

$\sum_{k=0}^{n} x^{k} W_{-2 k}=\frac{2 n(n+1) x^{n-1} W_{-2 n+2}+(n+1)((2 x-1) n+4 x) x^{n-1} W_{-2 n}}{4}$. 
(f) $(m=-2, j=1)$

$$
\text { If }(4 x-1)^{2} \neq 0 \text {, i.e., } x \neq \frac{1}{4} \text {, then }
$$

$\sum_{k=0}^{n} x^{k} W_{-2 k+1}=\frac{16 x^{n+1} W_{-2 n+3}+8(2 x-1) x^{n+1} W_{-2 n+1}+W_{1}-4\left(3 W_{1}-W_{0}\right) x}{(4 x-1)^{2}}$,

and

if $(4 x-1)^{2}=0$, i.e., $x=\frac{1}{4}$, then

$\sum_{k=0}^{n} x^{k} W_{-2 k+1}=\frac{2 n(n+1) x^{n-1} W_{-2 n+3}+(n+1)((2 x-1) n+4 x) x^{n-1} W_{-2 n+1}}{4}$.

From the above proposition, we have the following corollary which gives sum formulas of modified Oresme numbers (take $W_{n}=G_{n}$ with $G_{0}=0, G_{1}=1$ ).

Corollary 27. For $n \geq 0$, modified Oresme numbers have the following properties:

(a) $(m=1, j=0)$

$$
\text { If } \frac{1}{4}(x-2)^{2} \neq 0 \text {, i.e., } x \neq 2 \text {, then }
$$

$$
\sum_{k=0}^{n} x^{k} G_{k}=\frac{(x-4) x^{n+1} G_{n}+x^{n+1} G_{n-1}+4 x}{(x-2)^{2}},
$$

and

if $\frac{1}{4}(x-2)^{2}=0$, i.e., $x=2$, then

$$
\sum_{k=0}^{n} x^{k} G_{k}=\frac{(n+1)((x-4) n+2 x) x^{n-1} G_{n}+n(n+1) x^{n-1} G_{n-1}}{2} .
$$

(b) $(m=2, j=0)$

$$
\text { If } \frac{1}{16}(x-4)^{2} \neq 0 \text {, i.e., } x \neq 4 \text {, then }
$$

$$
\sum_{k=0}^{n} x^{k} G_{2 k}=\frac{(x-8) x^{n+1} G_{2 n}+x^{n+1} G_{2 n-2}+16 x}{(x-4)^{2}},
$$


and

if $\frac{1}{16}(x-4)^{2}=0$, i.e., $x=4$, then

$$
\sum_{k=0}^{n} x^{k} G_{2 k}=\frac{(n+1)((x-8) n+2 x) x^{n-1} G_{2 n}+n(n+1) x^{n-1} G_{2 n-2}}{2} .
$$

(c) $(m=2, j=1)$

If $\frac{1}{16}(x-4)^{2} \neq 0$, i.e., $x \neq 4$, then

$$
\sum_{k=0}^{n} x^{k} G_{2 k+1}=\frac{(x-8) x^{n+1} G_{2 n+1}+x^{n+1} G_{2 n-1}+4 x+16}{(x-4)^{2}},
$$

and

if $\frac{1}{16}(x-4)^{2}=0$, i.e., $x=4$, then

$\sum_{k=0}^{n} x^{k} G_{2 k+1}=\frac{(n+1)((x-8) n+2 x) x^{n-1} G_{2 n+1}+n(n+1) x^{n-1} G_{2 n-1}}{2}$.

(d) $(m=-1, j=0)$

If $(2 x-1)^{2} \neq 0$, i.e., $x \neq \frac{1}{2}$, then

$$
\sum_{k=0}^{n} x^{k} G_{-k}=\frac{4 x^{n+1} G_{-n+1}+4(x-1) x^{n+1} G_{-n}-4 x}{(2 x-1)^{2}},
$$

and

if $(2 x-1)^{2}=0$, i.e., $x=\frac{1}{2}$, then

$$
\sum_{k=0}^{n} x^{k} G_{-k}=\frac{n(n+1) x^{n-1} G_{-n+1}+(n+1)((x-1) n+2 x) x^{n-1} G_{-n}}{2} .
$$

(e) $(m=-2, j=0)$

If $(4 x-1)^{2} \neq 0$, i.e., $x \neq \frac{1}{4}$, then

$$
\sum_{k=0}^{n} x^{k} G_{-2 k}=\frac{16 x^{n+1} G_{-2 n+2}+8(2 x-1) x^{n+1} G_{-2 n}-16 x}{(4 x-1)^{2}},
$$


and

if $(4 x-1)^{2}=0$, i.e., $x=\frac{1}{4}$, then

$\sum_{k=0}^{n} x^{k} G_{-2 k}=\frac{2 n(n+1) x^{n-1} G_{-2 n+2}+(n+1)((2 x-1) n+4 x) x^{n-1} G_{-2 n}}{4}$.

(f) $(m=-2, j=1)$

If $(4 x-1)^{2} \neq 0$, i.e., $x \neq \frac{1}{4}$, then

$$
\sum_{k=0}^{n} x^{k} G_{-2 k+1}=\frac{16 x^{n+1} G_{-2 n+3}+8(2 x-1) x^{n+1} G_{-2 n+1}+1-12 x}{(4 x-1)^{2}},
$$

and

if $(4 x-1)^{2}=0$, i.e., $x=\frac{1}{4}$, then

$\sum_{k=0}^{n} x^{k} G_{-2 k+1}=\frac{2 n(n+1) x^{n-1} G_{-2 n+3}+(n+1)((2 x-1) n+4 x) x^{n-1} G_{-2 n+1}}{4}$.

Taking $W_{n}=H_{n}$ with $H_{0}=2, H_{1}=1$ in the last proposition, we have the following corollary which presents sum formulas of Oresme-Lucas numbers.

Corollary 28. For $n \geq 0$, Oresme-Lucas numbers have the following properties:

(a) $(m=1, j=0)$

If $\frac{1}{4}(x-2)^{2} \neq 0$, i.e., $x \neq 2$, then

$$
\sum_{k=0}^{n} x^{k} H_{k}=\frac{(x-4) x^{n+1} H_{n}+x^{n+1} H_{n-1}+8-4 x}{(x-2)^{2}},
$$

and

if $\frac{1}{4}(x-2)^{2}=0$, i.e., $x=2$, then

$$
\sum_{k=0}^{n} x^{k} H_{k}=\frac{(n+1)((x-4) n+2 x) x^{n-1} H_{n}+n(n+1) x^{n-1} H_{n-1}}{2} .
$$


(b) $(m=2, j=0)$

If $\frac{1}{16}(x-4)^{2} \neq 0$, i.e., $x \neq 4$, then

$$
\sum_{k=0}^{n} x^{k} H_{2 k}=\frac{(x-8) x^{n+1} H_{2 n}+x^{n+1} H_{2 n-2}+32-8 x}{(x-4)^{2}},
$$

and

if $\frac{1}{16}(x-4)^{2}=0$, i.e., $x=4$, then

$$
\sum_{k=0}^{n} x^{k} H_{2 k}=\frac{(n+1)((x-8) n+2 x) x^{n-1} H_{2 n}+n(n+1) x^{n-1} H_{2 n-2}}{2} .
$$

(c) $(m=2, j=1)$

If $\frac{1}{16}(x-4)^{2} \neq 0$, i.e., $x \neq 4$, then

$$
\sum_{k=0}^{n} x^{k} H_{2 k+1}=\frac{(x-8) x^{n+1} H_{2 n+1}+x^{n+1} H_{2 n-1}+16-4 x}{(x-4)^{2}},
$$

and

if $\frac{1}{16}(x-4)^{2}=0$, i.e., $x=4$, then

$$
\sum_{k=0}^{n} x^{k} H_{2 k+1}=\frac{(n+1)((x-8) n+2 x) x^{n-1} H_{2 n+1}+n(n+1) x^{n-1} H_{2 n-1}}{2} .
$$

(d) $(m=-1, j=0)$

If $(2 x-1)^{2} \neq 0$, i.e., $x \neq \frac{1}{2}$, then

$$
\sum_{k=0}^{n} x^{k} H_{-k}=\frac{4 x^{n+1} H_{-n+1}+4(x-1) x^{n+1} H_{-n}+2-4 x}{(2 x-1)^{2}},
$$

and

if $(2 x-1)^{2}=0$, i.e., $x=\frac{1}{2}$, then

$$
\sum_{k=0}^{n} x^{k} H_{-k}=\frac{n(n+1) x^{n-1} H_{-n+1}+(n+1)((x-1) n+2 x) x^{n-1} H_{-n}}{2} .
$$


(e) $(m=-2, j=0)$

$$
\begin{aligned}
& \text { If }(4 x-1)^{2} \neq 0 \text {, i.e., } x \neq \frac{1}{4} \text {, then } \\
& \qquad \sum_{k=0}^{n} x^{k} H_{-2 k}=\frac{16 x^{n+1} H_{-2 n+2}+8(2 x-1) x^{n+1} H_{-2 n}+2-8 x}{(4 x-1)^{2}},
\end{aligned}
$$

and

$$
\begin{aligned}
& \text { if }(4 x-1)^{2}=0, \text { i.e., } x=\frac{1}{4}, \text { then } \\
& \sum_{k=0}^{n} x^{k} H_{-2 k}=\frac{2 n(n+1) x^{n-1} H_{-2 n+2}+(n+1)((2 x-1) n+4 x) x^{n-1} H_{-2 n}}{4} .
\end{aligned}
$$

(f) $(m=-2, j=1)$

$$
\begin{aligned}
& \text { If }(4 x-1)^{2} \neq 0 \text {, i.e., } x \neq \frac{1}{4} \text {, then } \\
& \qquad \sum_{k=0}^{n} x^{k} H_{-2 k+1}=\frac{16 x^{n+1} H_{-2 n+3}+8(2 x-1) x^{n+1} H_{-2 n+1}+1-4 x}{(4 x-1)^{2}},
\end{aligned}
$$

and

$$
\begin{aligned}
& \text { if }(4 x-1)^{2}=0 \text {, i.e., } x=\frac{1}{4} \text {, then } \\
& \sum_{k=0}^{n} x^{k} H_{-2 k+1}=\frac{2 n(n+1) x^{n-1} H_{-2 n+3}+(n+1)((2 x-1) n+4 x) x^{n-1} H_{-2 n+1}}{4} .
\end{aligned}
$$

From the above proposition, we have the following corollary which gives sum formulas of Oresme numbers (take $W_{n}=O_{n}$ with $O_{0}=0, O_{1}=\frac{1}{2}$ ).

Corollary 29. For $n \geq 0$, Oresme numbers have the following properties:

(a) $(m=1, j=0)$

$$
\begin{aligned}
& \text { If } \frac{1}{4}(x-2)^{2} \neq 0 \text {, i.e., } x \neq 2 \text {, then } \\
& \qquad \sum_{k=0}^{n} x^{k} O_{k}=\frac{(x-4) x^{n+1} O_{n}+x^{n+1} O_{n-1}+2 x}{(x-2)^{2}},
\end{aligned}
$$


and

if $\frac{1}{4}(x-2)^{2}=0$, i.e., $x=2$, then

$$
\sum_{k=0}^{n} x^{k} O_{k}=\frac{(n+1)((x-4) n+2 x) x^{n-1} O_{n}+n(n+1) x^{n-1} O_{n-1}}{2} .
$$

(b) $(m=2, j=0)$

$$
\text { If } \frac{1}{16}(x-4)^{2} \neq 0 \text {, i.e., } x \neq 4 \text {, then }
$$

$$
\sum_{k=0}^{n} x^{k} O_{2 k}=\frac{(x-8) x^{n+1} O_{2 n}+x^{n+1} O_{2 n-2}+8 x}{(x-4)^{2}},
$$

and

if $\frac{1}{16}(x-4)^{2}=0$, i.e., $x=4$, then

$$
\sum_{k=0}^{n} x^{k} O_{2 k}=\frac{(n+1)((x-8) n+2 x) x^{n-1} O_{2 n}+n(n+1) x^{n-1} O_{2 n-2}}{2} .
$$

(c) $(m=2, j=1)$

$$
\text { If } \frac{1}{16}(x-4)^{2} \neq 0 \text {, i.e., } x \neq 4 \text {, then }
$$

$$
\sum_{k=0}^{n} x^{k} O_{2 k+1}=\frac{(x-8) x^{n+1} O_{2 n+1}+x^{n+1} O_{2 n-1}+2 x+8}{(x-4)^{2}},
$$

and

if $\frac{1}{16}(x-4)^{2}=0$, i.e., $x=4$, then

$$
\sum_{k=0}^{n} x^{k} O_{2 k+1}=\frac{(n+1)((x-8) n+2 x) x^{n-1} O_{2 n+1}+n(n+1) x^{n-1} O_{2 n-1}}{2} .
$$

(d) $(m=-1, j=0)$

$$
\text { If }(2 x-1)^{2} \neq 0 \text {, i.e., } x \neq \frac{1}{2} \text {, then }
$$

$$
\sum_{k=0}^{n} x^{k} O_{-k}=\frac{4 x^{n+1} O_{-n+1}+4(x-1) x^{n+1} O_{-n}-2 x}{(2 x-1)^{2}},
$$


and

if $(2 x-1)^{2}=0$, i.e., $x=\frac{1}{2}$, then

$$
\sum_{k=0}^{n} x^{k} O_{-k}=\frac{n(n+1) x^{n-1} O_{-n+1}+(n+1)((x-1) n+2 x) x^{n-1} O_{-n}}{2} .
$$

(e) $(m=-2, j=0)$

$$
\begin{aligned}
& \text { If }(4 x-1)^{2} \neq 0 \text {, i.e., } x \neq \frac{1}{4} \text {, then } \\
& \qquad \sum_{k=0}^{n} x^{k} O_{-2 k}=\frac{16 x^{n+1} O_{-2 n+2}+8(2 x-1) x^{n+1} O_{-2 n}-8 x}{(4 x-1)^{2}},
\end{aligned}
$$

and

$$
\begin{aligned}
& \text { if }(4 x-1)^{2}=0, \text { i.e., } x=\frac{1}{4}, \text { then } \\
& \sum_{k=0}^{n} x^{k} O_{-2 k}=\frac{2 n(n+1) x^{n-1} O_{-2 n+2}+(n+1)((2 x-1) n+4 x) x^{n-1} O_{-2 n}}{4} .
\end{aligned}
$$

(f) $(m=-2, j=1)$

$$
\begin{aligned}
& \text { If }(4 x-1)^{2} \neq 0, \text { i.e., } x \neq \frac{1}{4}, \text { then } \\
& \qquad \sum_{k=0}^{n} x^{k} O_{-2 k+1}=\frac{32 x^{n+1} O_{-2 n+3}+16(2 x-1) x^{n+1} O_{-2 n+1}+1-12 x}{2(4 x-1)^{2}},
\end{aligned}
$$

and

if $(4 x-1)^{2}=0$, i.e., $x=\frac{1}{4}$, then

$$
\sum_{k=0}^{n} x^{k} O_{-2 k+1}=\frac{2 n(n+1) x^{n-1} O_{-2 n+3}+(n+1)((2 x-1) n+4 x) x^{n-1} O_{-2 n+1}}{4} .
$$

Taking $x=1$ in the last three corollaries we get the following corollary.

Corollary 30. For $n \geq 0$, modified Oresme numbers, Oresme-Lucas numbers and Oresme numbers have the following properties: 
1.
(a) $\sum_{k=0}^{n} G_{k}=-3 G_{n}+G_{n-1}+4$.
(b) $\sum_{k=0}^{n} G_{2 k}=\frac{1}{9}\left(-7 G_{2 n}+G_{2 n-2}+16\right)$.
(c) $\sum_{k=0}^{n} G_{2 k+1}=\frac{1}{9}\left(-7 G_{2 n+1}+G_{2 n-1}+20\right)$.
(d) $\sum_{k=0}^{n} G_{-k}=4\left(G_{-n+1}-1\right)$.
(e) $\sum_{k=0}^{n} G_{-2 k}=\frac{8}{9}\left(2 G_{-2 n+2}+G_{-2 n}-2\right)$.
(f) $\sum_{k=0}^{n} G_{-2 k+1}=\frac{1}{9}\left(16 G_{-2 n+3}+8 G_{-2 n+1}-11\right)$.

2.
(a) $\sum_{k=0}^{n} H_{k}=-3 H_{n}+H_{n-1}+4$.
(b) $\sum_{k=0}^{n} H_{2 k}=\frac{1}{9}\left(-7 H_{2 n}+H_{2 n-2}+24\right)$.
(c) $\sum_{k=0}^{n} H_{2 k+1}=\frac{1}{9}\left(-7 H_{2 n+1}+H_{2 n-1}+12\right)$.
(d) $\sum_{k=0}^{n} H_{-k}=2\left(2 H_{-n+1}-1\right)$.
(e) $\sum_{k=0}^{n} H_{-2 k}=\frac{2}{9}\left(8 H_{-2 n+2}+4 H_{-2 n}-3\right)$.
(f) $\sum_{k=0}^{n} H_{-2 k+1}=\frac{1}{9}\left(16 H_{-2 n+3}+8 H_{-2 n+1}-3\right)$.

3.
(a) $\sum_{k=0}^{n} O_{k}=-3 O_{n}+O_{n-1}+2$.
(b) $\sum_{k=0}^{n} O_{2 k}=\frac{1}{9}\left(-7 O_{2 n}+O_{2 n-2}+8\right)$.
(c) $\sum_{k=0}^{n} O_{2 k+1}=\frac{1}{9}\left(-7 O_{2 n+1}+O_{2 n-1}+10\right)$.
(d) $\sum_{k=0}^{n} O_{-k}=2\left(2 O_{-n+1}-1\right)$.
(e) $\sum_{k=0}^{n} O_{-2 k}=\frac{8}{9}\left(2 O_{-2 n+2}+O_{-2 n}-1\right)$.
(f) $\sum_{k=0}^{n} O_{-2 k+1}=\frac{1}{18}\left(32 O_{-2 n+3}+16 O_{-2 n+1}-11\right)$. 


\section{Matrices Related with Generalized Oresme Numbers}

We define the square matrix $A$ of order 2 as:

$$
A=\left(\begin{array}{cc}
1 & -\frac{1}{4} \\
1 & 0
\end{array}\right)
$$

such that $\operatorname{det} A=\frac{1}{4}$. Then, we have

$$
\left(\begin{array}{c}
W_{n+1} \\
W_{n}
\end{array}\right)=\left(\begin{array}{cc}
1 & -\frac{1}{4} \\
1 & 0
\end{array}\right)\left(\begin{array}{c}
W_{n} \\
W_{n-1}
\end{array}\right)
$$

and

$$
\left(\begin{array}{c}
W_{n+1} \\
W_{n}
\end{array}\right)=\left(\begin{array}{cc}
1 & -\frac{1}{4} \\
1 & 0
\end{array}\right)^{n}\left(\begin{array}{l}
W_{1} \\
W_{0}
\end{array}\right) .
$$

If we take $W_{n}=G_{n}$ in 7.1 we have

$$
\left(\begin{array}{c}
G_{n+1} \\
G_{n}
\end{array}\right)=\left(\begin{array}{cc}
1 & -\frac{1}{4} \\
1 & 0
\end{array}\right)\left(\begin{array}{c}
G_{n} \\
G_{n-1}
\end{array}\right) .
$$

We also define

$$
B_{n}=\left(\begin{array}{cc}
G_{n+1} & -\frac{1}{4} G_{n} \\
G_{n} & -\frac{1}{4} G_{n-1}
\end{array}\right)
$$

and

$$
C_{n}=\left(\begin{array}{cc}
W_{n+1} & -\frac{1}{4} W_{n} \\
W_{n} & -\frac{1}{4} W_{n-1}
\end{array}\right) .
$$

Theorem 31. For all integers $m, n$, we have

(a) $B_{n}=A^{n}$

(b) $C_{1} A^{n}=A^{n} C_{1}$

(c) $C_{n+m}=C_{n} B_{m}=B_{m} C_{n}$.

Proof. Take $r=1, s=-\frac{1}{4}$ in Soykan 12, Theorem 5.1.]. 
Corollary 32. For all integers n, we have the following formulas for the modified Oresme, Oresme-Lucas and Oresme numbers.

(a) Modified Oresme Numbers.

$$
A^{n}=\left(\begin{array}{cc}
1 & -\frac{1}{4} \\
1 & 0
\end{array}\right)^{n}=\left(\begin{array}{cc}
G_{n+1} & -\frac{1}{4} G_{n} \\
G_{n} & -\frac{1}{4} G_{n-1}
\end{array}\right) .
$$

(b) Oresme-Lucas Numbers.

$$
A^{n}=\left(\begin{array}{cc}
1 & -\frac{1}{4} \\
1 & 0
\end{array}\right)^{n}=\left(\begin{array}{cc}
(n+1) H_{n+1} & -\frac{1}{4} n H_{n} \\
n H_{n} & -\frac{1}{4}(n-1) H_{n-1}
\end{array}\right) .
$$

(c) Oresme Numbers.

$$
A^{n}=\left(\begin{array}{cc}
1 & -\frac{1}{4} \\
1 & 0
\end{array}\right)^{n}=\left(\begin{array}{cc}
2 O_{n+1} & -\frac{1}{2} O_{n} \\
2 O_{n} & -\frac{1}{2} O_{n-1}
\end{array}\right) .
$$

Proof. (a) It is given in Theorem 31 (a).

(b) Note that, from Lemma 14 , we have

$$
G_{n}=n H_{n}
$$

Using the last equation and (a), we get required result.

(c) Note that, from Lemma 14, we have

$$
G_{n}=2 O_{n}
$$

Using the last equation and (a), we get required result.

Theorem 33. For all integers $m, n$, we have

$$
W_{n+m}=W_{n} G_{m+1}-\frac{1}{4} W_{n-1} G_{m}
$$

Proof. Take $r=1, s=-\frac{1}{4}$ in Soykan 12 , Theorem 5.2.]. 
By Lemma 11, we know that

$$
\left(W_{0}-2 W_{1}\right)^{2} G_{m}=-4 W_{0} W_{m+1}+4 W_{1} W_{m},
$$

so 7.3 can be written in the following form

$$
\left(W_{0}-2 W_{1}\right)^{2} W_{n+m}=W_{n}\left(4\left(W_{1}-W_{0}\right) W_{m+1}+W_{0} W_{m}\right)+W_{n-1}\left(W_{0} W_{m+1}-W_{1} W_{m}\right) .
$$

Corollary 34. For all integers $m, n$, we have

$$
\begin{aligned}
G_{n+m} & =G_{n} G_{m+1}-\frac{1}{4} G_{n-1} G_{m}, \\
H_{n+m} & =H_{n} G_{m+1}-\frac{1}{4} H_{n-1} G_{m}, \\
O_{n+m} & =O_{n} G_{m+1}-\frac{1}{4} O_{n-1} G_{m},
\end{aligned}
$$

and

$$
O_{n+m}=2 O_{n} O_{m+1}-\frac{1}{2} O_{n-1} O_{m}
$$

\section{References}

[1] M. Clagett, Nicole Oresme and the Medieval Geometry of Qualities and Motions: A Treatise on the Uniformity and Difformity of Intensities Known as Tractatus de confurationibus qualitatum et motuum, The University of Wisconsin Press, Wisconsin, 1968.

[2] M. Clagett, "Oresme, Nicole", in: C.C. Gillespie (ed.), Dictionary of Scientific Biography, Vol. 9, Charles Scribner's Sons, New York, 1981, pp. 223-230.

[3] C.K. Cook, Some sums related to sums of Oresme numbers, in: Howard F. T. (eds.) Applications of Fibonacci Numbers, volume 9, Proceedings of the Tenth International Research Conference on Fibonacci Numbers and Their Applications, Kluwer Academic Publishers, 2004, pp. 87-99.

https://doi.org/10.1007/978-0-306-48517-6_10

[4] A.F. Horadam, Oresme numbers, Fibonacci Quarterly 12(3) (1974), 267-271.

[5] A.F. Horadam, A generalized Fibonacci sequence, American Mathematical Monthly 68 (1961), 455-459. https://doi.org/10.1080/00029890.1961.11989696 
[6] A.F. Horadam, Basic properties of a certain generalized sequence of numbers, Fibonacci Quarterly 3.3 (1965), 161-176.

[7] A.F. Horadam, Special properties of the sequence $w_{n}(a, b ; p, q)$, Fibonacci Quarterly $5(5)$ (1967), 424-434.

[8] A.F. Horadam, Generating functions for powers of a certain generalized sequence of numbers, Duke Math. J. 32 (1965), 437-446.

https://doi.org/10.1215/S0012-7094-65-03244-8

[9] M.C.S. Mangueira, R.P.M. Vieira, F.R.V. Alves and P.M.M.C. Catarino, The Oresme sequence: The generalization of its matrix form and its hybridization process, Notes on Number Theory and Discrete Mathematics 27(1) (2021), 101-111. https://doi.org/10.7546/nntdm.2021.27.1.101-111

[10] N.J.A. Sloane, The on-line encyclopedia of integer sequences, http://oeis.org/

[11] Y. Soykan, Simson identity of generalized m-step Fibonacci numbers, Int. J. Adv. Appl. Math. Mech. 7(2) (2019), 45-56.

[12] Y. Soykan, On generalized (r,s)-numbers, Int. J. Adv. Appl. Math. Mech. 8(1) (2020), $1-14$.

[13] Y. Soykan, Some properties of generalized Fibonacci numbers: identities, recurrence properties and closed forms of the sum formulas $\sum_{k=0}^{n} x^{k} W_{m k+j}$, Archives of Current Research International 21(3) (2021), 11-38.

https://doi.org/10.9734/acri/2021/v21i330235

This is an open access article distributed under the terms of the Creative Commons Attribution License (http://creativecommons.org/licenses/by/4.0/), which permits unrestricted, use, distribution and reproduction in any medium, or format for any purpose, even commercially provided the work is properly cited. 